\title{
Is there a relation between computed tomography findings and electrocardiography findings in COVID-19?

\author{
Fatih Öztürk ${ }^{1 *} \odot$, Naci Babat ${ }^{\circledR} \odot$, Cemil Göya ${ }^{2} \odot$, Saim Türkoğlu² $\oplus^{\oplus}$,

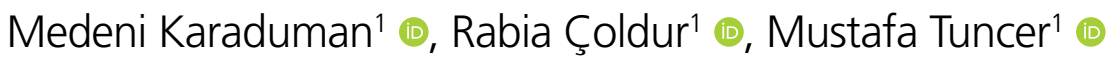

\begin{abstract}
SUMMARY
OBJECTIVE: COVID-19 can cause lung damage and may present with pneumonia in patients. In the present study, the correlation between the severity of pneumonia and electrocardiography parameters of COVID-19 were examined.

METHODS: A total of 93 COVID-19 patients and a control group consisting of 62 volunteers were studied. Computed thorax tomography evaluation was performed; each lung was divided into three zones. For each affected zone, scores were given. The main computed thorax tomography patterns were described in line with the terms defined by the Fleischner Society and peer reviewed literature on viral pneumonia. We compared Computed thorax tomography of patients with corrected QT (QTC) and P wave dispersion (Pd) time.

RESULTS: There is a significant difference between the patient and control groups in terms of QTC values ( $413.5 \pm 28.8 \mathrm{msec}$ vs. $395.6 \pm 16.7$ msec $p<0.001$ ). Likewise, the Pd value of the patient group is statistically significantly higher than that of the control group ( $50.0 \pm 9.6$ ms computed thorax tomography ec vs. $41.3 \pm 5.8 \mathrm{msec} p<0.001)$. In the patient group, a reverse correlation was detected between computed thorax tomography score and Pd value according to partial correlation coefficient analysis (correlation coefficient: -0.232 , $\mathrm{p}=0.027$ ). In the patient group, the correlation between computed thorax tomography score and QTC value was similarly determined according to partial correlation coefficient analysis (Correlation coefficient:0.224, $\mathrm{p}=0.017$ ).
\end{abstract}

CONCLUSIONS: COVID-19 prolongs QTC and P wave dispersion values; and as the severity of pneumonia increases, QTC value increases. However, whereas the severity of pneumonia increases, $\mathrm{P}$ wave dispersion value decreases.

KEYWORDS: Coronavirus infections. Electrocardiography. Tomography, X-Ray Computed.

\section{INTRODUCTION}

The COVID-19 disease first appeared in Wuhan, province in China, in December 2019. It quickly spread to other regions of China and other countries, and caused a pandemic ${ }^{1}$. COVID19 has the feature to affect many organs, especially the lungs, but lung involvement and pneumonia are among the most important mortalities ${ }^{2}$. Reverse transcription polymerase chain reaction and computed tomography $(\mathrm{CT})$ are generally used in diagnosis ${ }^{3}$. In this manner, pneumonia can be interpreted with CT. CT is frequently used in treatment follow-up and prognosis ${ }^{4}$. Aside from the lungs, the heart is also one of the affected organs, presenting with symptoms such as arrhythmia, heart muscle damage, and heart failure ${ }^{5}$. In a study, the QT duration in electrocardiography (ECG) of COVID-19 patients

'Van Yüzüncü Yıl Universty, Cardiyology Department - Van, Turkey.

${ }^{2}$ Van Yüzüncü Yll Universty, Radiology Department - Van, Turkey.

*Corresponding author: fatihozturk2488@gmail.com

Conflicts of interest: the authors declare there are no conflicts of interest. Funding: none.

Received on October 13, 2020. Accepted on November 08, 2020. 
was shown to be longer than that of populations without the disease $^{6}$. Furthermore, drugs used in COVID-19 treatment prolong QT duration ${ }^{7}$. There is no study examining the correlation between COVID-19 pneumonia and QT interval. In addition, diseases affecting the lungs are known to have an effect on the atrium and thus the $\mathrm{P}$ wave in $\mathrm{ECG}^{8,9}$. It is possible that COVID-19 causes pneumonia, which increases the volume load in the atrium and affects the $\mathrm{P}$ wave. The condition of the atrium can be estimated by the dispersion of the $\mathrm{p}$ wave $(\mathrm{Pd})$ in $\mathrm{ECG}^{8,9}$. In this study, the correlation between the lung involvement severity in COVID-19 patients and $\mathrm{Pd}$ and QT was examined.

\section{METHODS}

A total of 93 COVID-19 patients and a control group consisting of 62 volunteers who applied in our hospital from April to July 2020 were enrolled. The study was approved by the Local Medical Ethics Committee of Van Yuzuncu Yil University. Positive Polymerase chain reaction (PCR) test for COVID-19 was identified as the inclusion criteria.

Patients with pregnancy, chronic kidney failure, chronic liver failure, malignancy, coronary artery disease, heart failure, and heart valve disease were excluded from the study. Moreover, patients with a known rhythm disorder (atrial fibrillation, subraventricular tachycardia, Wolf Parkinson White, ventricular rhythm disorders, etc.) or a pathological (pathological Q, T negativity, ST changes etc.) ECG findings were excluded.

12-lead ECG records (Nihon Kohden brand) were received after 30 minutes of rest, at room temperature $\left(20-24^{\circ} \mathrm{C}\right)$. All ECGs (filter range $0.5 \mathrm{~Hz}$ to $150 \mathrm{~Hz}$, AC filter $60 \mathrm{~Hz}$, $25 \mathrm{~mm} / \mathrm{s}, 10 \mathrm{~mm} / \mathrm{mV}$ ) were analyzed by two independent clinicians, who were blinded to the study design and clinical data. All ECG records were scanned, the data was transferred to a personal computer to reduce possible errors, and Adobe Photoshop software was used for $400 \%$ magnification. The participants having $U$ wave in their ECGs were not included in the study. For each lead, three values were calculated averagely. The duration from the beginning of the $\mathrm{P}$ wave to the end were measured in all leads. Pd value was calculated by subtracting the Pmin width from the Pmax width QT interval, measured from the beginning of the QRS complex to the end of the $\mathrm{T}$ wave; corrected QT interval was calculated by using Bazett formula $(\mathrm{QT} c=\mathrm{QT} \sqrt{ }(\mathrm{R}-\mathrm{R}$ interval $))$.

Non-contrast, $3 \mathrm{~mm}$ slice thickness CT findings of the patients with COVID-19 pneumonia pre-diagnosis and positive PCR test were evaluated.

The CT images were taken using a 16-slice multidetector CT (MDCT) device (Somatom Emotion 16-slice;
CT2012E- Siemens AG, Berlin and Munich, Germany). CT evaluation was done by two independent radiologists. Briefly, each lung was divided into three zones: upper (above the carina), middle, and lower (below the inferior pulmonary vein) zones. For each affected zone, scores were given. The main CT patterns were described in line with the terms defined by the Fleischner Society and peer-reviewed literature on viral pneumonia ${ }^{10,11}$.

Typical CT findings are ground-glass opacification $(G G O)$, air bronchogram, traction bronchiectasis, subpleural atelectasis, and peribroncovascular thickening; atypical findings are mediastinal lymph node, pleural effusion, tree-in bud, pneumothorax and cavitation.

As to the findings, 3 points for ground-glass opacity, 2 for peripheral-subpleural involvement, 2 for consolidation, and 2 for air bronchogram. Besides that, centrilobuler nodular opacity, subpleural atelectasis, halo-reverse halo, air buble sign were evaluated, and 1 point was given for each finding.

Pleural-pericardial effusion and mediastinal lymph node are atypical findings with no score.

The impact rate is multiplied by 1 if the lung involvement rate is $0-25 \%$, by 2 if ti is $26-50 \%$, by 3 if it is $51-75 \%$, and by 4 if it is more than $76 \%$. (Figure 1 ).

For example, the patient has involvement in the upper right zone, it is 1 point For ground-glass opacity; 3 points are given; $40 \%$ of all zones is affected; $(1+3) \chi^{2}=8$ points were calculated (Figure 1).

CT and ECG examinations of the participants were performed before treatment.

Echocardiography examination was conducted in all patients. The Echocardiography examination was performed at least 15 min after the rest using a Vivid E9 (Vivid 9 Pro, General Electric Medical Systems, Milwaukee, Wisconsin, USA) device and an X5-1 transthoracic probe in the left lateral position (two-dimensional, M-mode, color Doppler echocardiography)

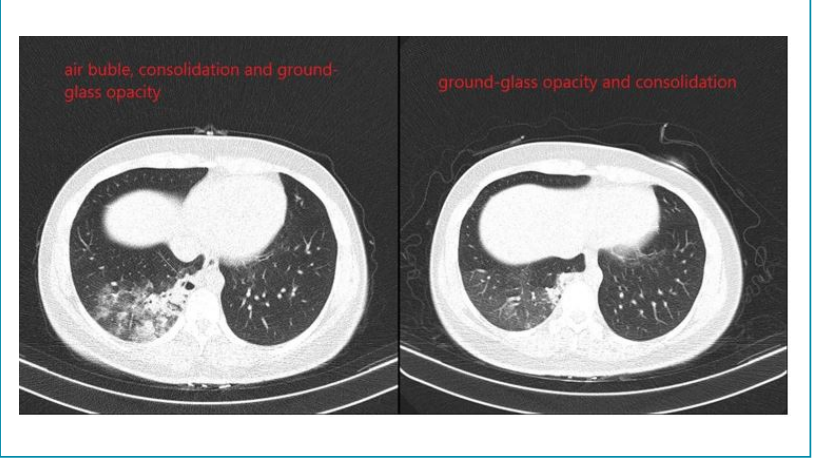

Figure 1. Examples of ground glass opacity, air bubble and consolidation. 
using parasternal and apical windows. All the Echocardiography examinations were performed in accordance with the American Society of Echocardiography (ASE) guidelines and the European Standard Echocardiography Guidelines ${ }^{12}$.

\section{Statıstical analysıs}

Whereas descriptive statistics for continuous variables are expressed as average and standard deviation, it is expressed as numbers and percentages for categorical variables. Student t test was used in comparison of groups according to continuous variables. The group and categorical variables were compared using $\chi^{2}$ test. Partial correlation coefficient was calculated considering Pd and age in determining the correlation between QTc and CT Score values, likewise between Pd and CT score values. In calculations, 5\% was considered statistically significant, and all statistical analyses were performed using SPSS (ver. 22.0) statistical package programme.

\section{RESULTS}

The clinical and demographic characteristics of patients and control group are shown in Table 1 . There is a statistically significant difference in QTc values between patient and control groups (413.5 \pm 28.8 msec vs. 395.6 \pm 16.7 msec $p<0.001$ ). Similarly, the $\mathrm{Pd}$ value of the patient group is statistically significantly higher than the control group's Pd value $(50.0 \pm 9.6$ msec vs. $41.3 \pm 5.8$ msec $\mathrm{p}<0.001)$. Between the patient and the control group, age, gender, Hypertension (HT), Diabetes Mellitus (DM), and Ejection Fraction (EF) parameters are not different significantly.

In the patient group, a reverse correlation was detected between CT score and Pd value according to Partial correlation coefficient analysis (Correlation coefficient: -0.232, p=0.027). $\mathrm{Pd}$ value of the patients with higher scores were calculated lower.
The correlation between CT score and QTc value was similarly determined according to Partial correlation coefficient analysis in the patient group (Correlation coefficient: 0.224, $\mathrm{p}=0.017$ ). Patients with high scores also had a high QTc value (Figure 2). No statistically significant correlation was detected between CT score and gender, HT, DM, EF in the patient group.

In addition to this, the correlation between the categories of QTc, Pd and score variables is shown visually in Figure 2. 30-44 msec category of Pd, 6-56 categories of the score value and 397-431 mseccategories of QTc are indicated in Figure 2, in the positive region according to the first dimension. It is expected

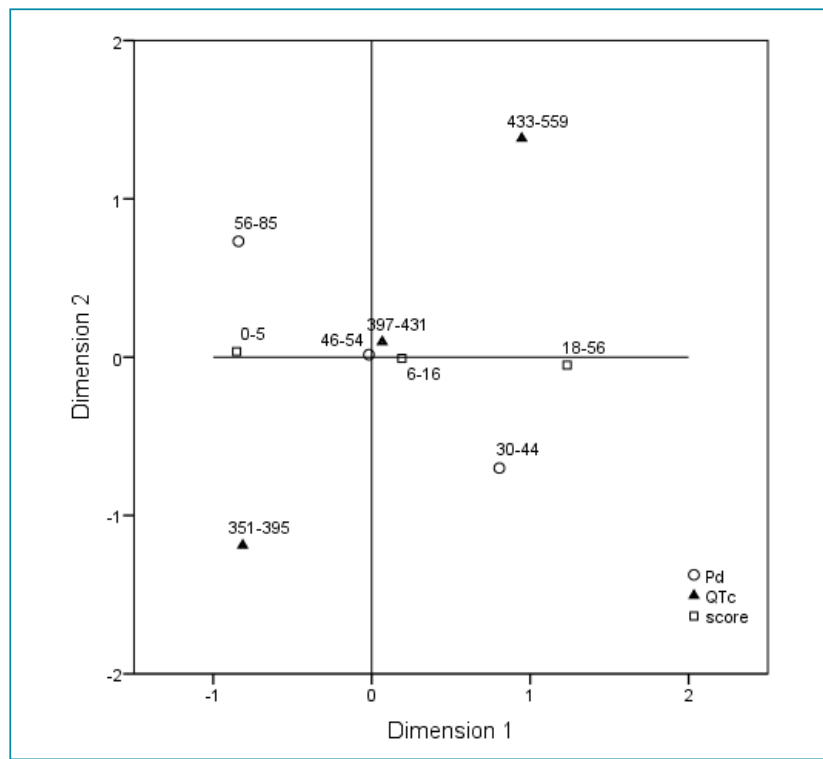

Figure 2. Configuration of the $\mathrm{P}$ wave dispersion, QTC and score on two dimensional map, categorical principal component analysis.

Table 1. Descriptive statistics and comparison results for clinical and demographic characteristics.

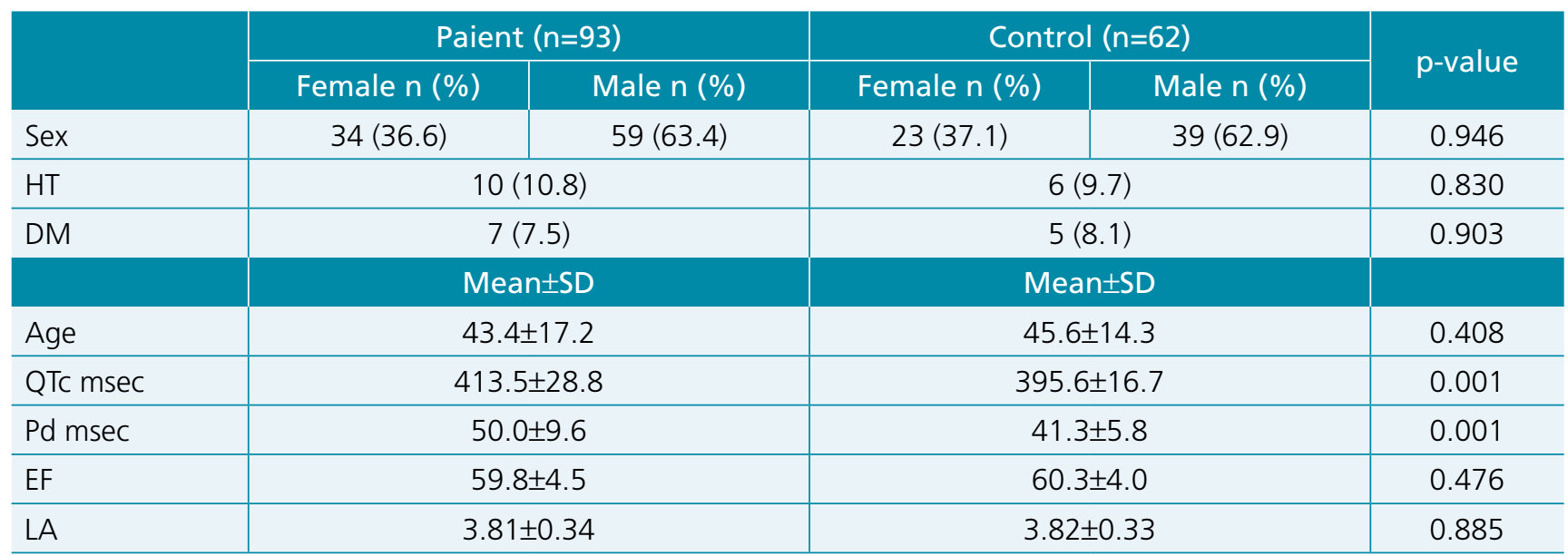

HT: hypertension; DM: diabetes mellitus; QTc: corrected QT; SD: standard deviation; Pd: p wave dispersion; EF: ejection fraction; LA: left atrium. 
that the score values should be between 6-56 in individuals with a QTc of 397-431 msec and a Pd in the range of 30-44 msec. Similarly, in Figure 2, in the negative region according to the first dimension, 56-85 msec of Pd, 351-395 msec of QTc and $0-5$ categories of score value are shown and according to this, the score value is expected to be in the range of $0-5$ in patients with a QTc value of 351-395 msec and a Pd value of 55-86 msec.

Moreover, there is no statistically significant relation between the right atrium and $P d(p=0.572)$. Similiarly, we did not find a significant relation between troponin value, $\mathrm{Pd}$ and QTc $(\mathrm{p}=0.125, \mathrm{p}=0.181$, respectively)

\section{DISCUSSION}

Prolonged QTc and Pd values in COVID-19 patients were detected. A positive correlation between the degree (severity) of the lung involvement and the QTc value was also found, whereas the reverse correlation between the degree (severity) of the lung involvement and the Pd value was determined. These findings may be an important guide for approach and treatment in COVD-19.

COVID-19 was shown to cause cardiac arrhythmia ${ }^{13}$. Additionally, studies show that it causes ventricular arrhythmia and prolongs the QTc duration, being the predictor of ventricular arrhythmia ${ }^{6,14}$. There are also case reports about the correlation between COVID-19 and atrial arrhythmias ${ }^{15}$. Pd is used as an indicator for atrial arrhythmias (especially atrial fibrillation) ${ }^{16}$. In the present study, we found higher Pd and QTc values in the patient group. These values were calculated before treatment; in the given circumstances, COVID-19 prolonged the duration of $\mathrm{Pd}$ and QTc, regardless of the treatment. The fact that Pd is an indicator for atrial fibrillation (AF) and the high Pd value in COVID-19 shows COVID-19 may be a predictor for the development of AF.

Furthermore, autonomic system, endothelial dysfunction, and inflammatory response have an impact on $\mathrm{p}$ wave duration and dispersion ${ }^{17,18}$. The fact that autonomic imbalance affects intra-atrial and interatrial conduction times may be prone to atrial arrhythmias ${ }^{19}$. The virus SARS-CoV-2, the virus that causes COVID-19, had neurotropic feature and involvement of the brainstem cardiorespiratory nuclei ${ }^{20}$. CT has an important role in guiding the diagnosis and treatment of COVID$19^{21}$. Various scores were used to determine the severity of COVID-19 pneumonia with lung findings in CT; severity of the disease tried to be determined ${ }^{22}$. There is no broad-scale research conducted between CT findings of lung involvement and ECG parameters. In the present study, patients with high lung involvement severity had high QTc values. The fact that lung involvement also affects the conduction system of heart and impairs the ventricular repolarization phase are the indicator for increasing potential of ventricular arrhythmias (ventricular tachycardia and ventricular fibrillation).

Among the causes of cardiac arrhythmias, hormonal disorders, electrolyte imbalances, myocardial cell damage, adrenergic stress, and inflammation are shown. COVID-19 can also cause these pathologies ${ }^{23}$. Even though studies examining COVID19 in terms of ventricular arrhythmias are available in the literature, there are not enough studies on atrial arrhythmias. In the present study, the correlation between Pd value and pneumonia severity as well as Pd value impaired due to COVID19 were shown. The reverse change in the lung involvement of COVID-19 and Pd value shows that the mechanism of the disease affecting atrial conduction and causing pneumonia is different. The high Pd value of patients without severe pneumonia means that they are more prone to atrial arrhythmias, such as atrial fibrillation ${ }^{24}$. This situation indicates that patients tend to suffer with thromboembolic events in the future ${ }^{25}$.

Our study is not sufficient to answer the question why Pd is less affected in severe pneumonia. Many factors may be discussed and large scaled studies are needed in this field.

\section{Limitations}

Among the limitations of the present study, the relative small sample size is one of them. The hormonal conditions of patients affect the ECG parameters, and no evaluation of hormones was performed herein, which is another limitation. The effect of hormone values on the ECG parameters is known, and the fact that no evaluation of hormones was made is one of the limitations of present study. Since the pathophysiology of COVID19 is not clear, trying to explain the mechanism affecting ECG parameters is not enough, another limitation.

\section{CONCLUSIONS}

As a result, COVID-19 prolongs the QTc and Pd values. Whereas the severity of pneumonia increases in COVID-19, QTc is prolonged. Pd value, which is another ECG finding, decreases as the severity of pneumonia increases.

\section{AUTHORS" CONTRIBUTIONS}

FÖ: Conceptualization, Data Curation, Formal Analysis, Writing - Original Draft, Writing - Review \& Editing. MT: Conceptualization, Data Curation, Writing - Review \& Editing. CG: Conceptualization, Data Curation, Writing - Review \& Editing. ST: Conceptualization, Formal Analysis. MD: Data Curation, Formal Analysis, Writing - Original Draft. RÇ: Data Curation, Formal Analysis, Writing - Review \& Editing, NB: Data Curation, Writing-Original Draft, Writing - Review \& Editing. 


\section{REFERENCES}

1. Li Q, Guan X, Wu P, Wang X, Zhou L, Tong Y, et al. Early transmission dynamics in Wuhan, China, of novel coronavirusinfected pneumonia. N Engl J Med. 2020;382(13):1199-207. https://doi.org/10.1056/NEJMoa2001316

2. Abbasi-Oshaghi E, Mirzaei F, Farahani F, Khodadadi I, Tayebinia $\mathrm{H}$. Diagnosis and treatment of coronavirus disease 2019 (COVID-19): laboratory, PCR, and chest CT imaging findings. Int J Surg. 2020;79:143-53. https://doi.org/10.1016/j. ijsu.2020.05.018

3. Huang $P$, Liu T, Huang L, Liu H, Lei $M, X u W$, et al. Use of chest CT in combination with negative RT-PCR assay for the 2019 novel coronavirus but high clinical suspicion. Radiology. 2020;295(1):22-3. https://doi.org/10.1148/radiol.2020200330

4. Fang $Y$, Zhang $H$, Xie J, Lin $M$, Ying $L$, Pang $P$, et al. Sensitivity of chest CT for COVID-19: comparison to RT-PCR. Radiology. 2020;296(2):E115-7. https://doi.org/10.1148/ radiol.2020200432

5. Guzik TJ, Mohiddin SA, Dimarco A, Patel V, Savvatis K, MarelliBerg FM, et al. COVID-19 and the cardiovascular system: implications for risk assessment, diagnosis, and treatment options. Cardiovasc Res. 2020;116(10):1666-87. https://doi. org/10.1093/cvr/cvaa106

6. Öztürk F, Karaduman M, Çoldur R, İncecik Ş, Güneş Y, Tuncer $M$. Interpretation of arrhythmogenic effects of COVID-19 disease through ECG. Aging Male. 2020;23(5):1362-5. https:// doi.org/10.1080/13685538.2020.1769058

7. Zhou D, Dai SM, Tong Q. COVID-19: a recommendation to examine the effect of hydroxychloroquine in preventing infection and progression. J Antimicrob Chemother. 2020;75(7):166770. https://doi.org/10.1093/jac/dkaa114

8. Medford ARL. Arrhythmias in COPD: consider P-wave dispersion and pulmonary hypertension, too. Chest. 2013;143(2):57980. https://doi.org/10.1378/chest.12-2199

9. Okutucu S, Aytemir K, Oto A. P-wave dispersion: what we know till now? JRSM Cardiovasc Dis. 2016;5:2048004016639443. https://doi.org/10.1177/2048004016639443

10. Hansell DM, Bankier AA, MacMahon H, McLoud TC, Müller NL, Remy J. Fleischner Society: glossary of terms for thoracic imaging. Radiology. 2008;246(3):697-722. https://doi. org/10.1148/radiol.2462070712

11. Ooi GC, Khong PL, Müller NL, Yiu WC, Zhou LJ, Ho JC, et al. Severe acute respiratory syndrome: temporal lung changes at thin-section CT in 30 patients. Radiology. 2004;230(3):836-44. https://doi.org/10.1148/radiol.2303030853

12. Lancellotti P, Tribouilloy C, Hagendorff A, Popescu BA, Edvardsen T, Pierard LA, et al. Recommendations for the echocardiographic assessment of native valvular regurgitation: an executive summary from the European Association of Cardiovascular Imaging. Eur Heart J Cardiovasc Imaging. 2013;14(7):611-44. https://doi.org/10.1093/ehjci/jet105

13. Wang D, Hu B, Hu C, Zhu F, Liu X, Zhang J, et al. Clinical characteristics of 138 hospitalized patients with 2019 novel coronavirus-infected pneumonia in Wuhan, China.
Jama. 2020;323(11):1061-9. https://doi.org/10.1001/ jama.2020.1585

14. Guo T, Fan Y, Chen M, Wu X, Zhang L, He T, et al. Cardiovascular implications of fatal outcomes of patients with coronavirus disease 2019 (COVID-19). JAMA Cardiol. 2020;5(7):811-8. https://doi.org/10.1001/jamacardio.2020.1017

15. Seecheran R, Narayansingh R, Giddings S, Rampaul M, Furlonge $\mathrm{K}$, Abdool $\mathrm{K}$, et al. Atrial arrhythmias in a patient presenting with coronavirus disease-2019 (COVID-19) infection. J Investig Med High Impact Case Rep. 2020;8:2324709620925571. https://doi.org/10.1177/2324709620925571

16. Guntekin U, Gunes Y, Simsek H, Tuncer M, Arslan S. P wave duration and dispersion in patients with hyperthyroidism and the short-term effects of antithyroid treatment. Indian Pacing Electrophysiol J. 2009;9(5):251-9. PMID: 19763193

17. Yasar E, Yilmaz B, Yasar AS, Goktepe AS, Alaca R, Mohur $\mathrm{H}$. Effect of autonomic dysfunction on $\mathrm{p}$-wave dispersion in patients with chronic spinal cord injury. Am J Phys Med Rehabil. 2010;89(10):824-30. https://doi.org/10.1097/ PHM.0b013e3181f1ba2c

18. Conlon R, Tanner R, David S, Szeplaki G, Galvin J, Keaney J, et al. Evaluation of the Tp-Te interval, QTc and P-wave dispersion in patients with coronary artery ectasia. Cardiol Res. 2017;8(6):280-5. https://doi.org/10.14740/cr631w

19. Kosar F, Aksoy Y, Ari F, Keskin L, Sahin I. P-wave duration and dispersion in obese subjects. Ann Noninvasive Electrocardiol. 2008;13(1):3-7. https://doi.org/10.1111/j.1542474X.2007.00194.x

20. Li YC, Bai WZ, Hashikawa T. The neuroinvasive potential of SARS-CoV2 may play a role in the respiratory failure of COVID-19 patients. J Med Virol. 2020;92(6):552-5. https:// doi.org/10.1002/jmv.25728

21. Kanne JP. Chest CT findings in 2019 novel coronavirus (2019-nCoV) infections from Wuhan, China: key points for the radiologist. Radiology. 2020;295(1):16-7. https://doi. org/10.1148/radiol.2020200241

22. Wang Y, Dong C, Hu Y, Li C, Ren Q, Zhang X, et al. Temporal changes of CT findings in 90 patients with COVID-19 pneumonia: a longitudinal study. Radiology. 2020;296(2):E5564. https://doi.org/10.1148/radiol.2020200843

23. Boukhris M, Hillani A, Moroni F, Annabi MS, Addad F, Ribeiro MH, et al. Cardiovascular implications of the COVID-19 pandemic: a global perspective. Can J Cardiol. 2020;36(7):1068-80. https://doi.org/10.1016/j.cjca.2020.05.018

24. Rosiak M, Bolinska $H$, Ruta J. P wave dispersion and $P$ wave duration on SAECG in predicting atrial fibrillation in patients with acute myocardial infarction. Ann Noninvasive Electrocardiol. 2002;7(4):363-8. https://doi.org/10.1111/j.1542-474x.2002. tb00186.x

25. Procter NE, Stewart S, Horowitz JD. New-onset atrial fibrillation and thromboembolic risk: cardiovascular syzygy? Heart Rhythm. 2016;13(6):1355-61. https://doi.org/10.1016/j. hrthm.2015.12.010 